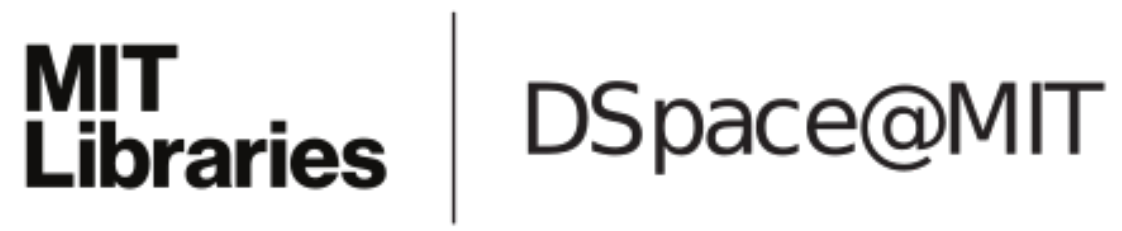

\author{
MIT Open Access Articles
}

\section{A digitally-assisted sensor interface for biomedical applications}

The MIT Faculty has made this article openly available. Please share how this access benefits you. Your story matters.

Citation: Bohorquez, Jose L. et al. “A Digitally-assisted Sensor Interface for Biomedical Applications." Technical Digest of Technical Papers, 2010 IEEE Symposium on VLSI Circuits (VLSIC), IEEE, 2010. 217-218. Web.C 2010 IEEE.

As Published: http://dx.doi.org/10.1109/VLSIC.2010.5560293

Publisher: Institute of Electrical and Electronics Engineers

Persistent URL: http://hdl.handle.net/1721.1/71833

Version: Final published version: final published article, as it appeared in a journal, conference proceedings, or other formally published context

Terms of Use: Article is made available in accordance with the publisher's policy and may be subject to US copyright law. Please refer to the publisher's site for terms of use. 


\title{
A Digitally-Assisted Sensor Interface for Biomedical Applications
}

\author{
Jose L. Bohorquez, Marcus Yip, Anantha P. Chandrakasan, and Joel L. Dawson * \\ Massachusetts Institute of Technology, Cambridge, MA 02139, USA
}

\begin{abstract}
A compact, low-power, digitally-assisted sensor interface for biomedical applications is presented. It exploits oversampling and digital design to reduce system area and power, while making the system more robust to interferers. Anti-aliasing is achieved using a charge-sampling filter with a sinc frequency response and programmable gain. A mixed-signal feedback loop creates a sharp, programmable notch for interference cancelation. A prototype was implemented in $0.18 \mu \mathrm{m} \mathrm{CMOS} \mathrm{and}$ the on-chip blocks consume a total of $255 \mathrm{nW}-2.5 \mu \mathrm{W}$ from a $1.5 \mathrm{~V}$ supply depending on noise and bandwidth requirements.
\end{abstract}

\section{Introduction}

Electrocardiograms (ECG), electroencephalograms (EEG), and electromyograms (EMG) are common bio-potential signals measured non-invasively. Neural field potentials, in contrast, are measured using medical implants $[1,2]$. In most cases, an analog front-end (AFE) comprising an instrumentation amplifier (IAMP) and an anti-aliasing filter is used to process these signals. They can be as small as a few $\mu \mathrm{V}$ 's and typically reside somewhere between $1 \mathrm{~Hz}$ to $1 \mathrm{kHz}$.

Minimizing power consumption in implants is critical since batteries must be small and last 5-10 years. This is particularly important for applications that use multi-electrode arrays with tens or hundreds of sensors, such as brain-machine interfaces. Since the electrode pitch can be as small as $400 \mu \mathrm{m}$, each AFE must also be very small [1]. For non-invasive devices that use hundreds of sensors, such as modern EEG systems, there is a financial incentive to minimize area and power, particularly if consumer products are targeted.

There are four main types of aggressors that corrupt biopotential signals: electrode DC offset (EDO), IAMP flicker noise, IAMP thermal noise, and $50 / 60 \mathrm{~Hz}$ power line interference (PLI). AFEs presented in recent literature achieve very low thermal noise and minimize the effects of EDO while consuming minimal power $[1,2]$. Further, the effects of flicker noise are minimized in [2] by using chopping. Most AFE implementations try to mitigate the effects of common-mode PLI by using an IAMP with a high input impedance. This is often insufficient, however, since some PLI is converted to differentialmode interference as displacement currents flow through finite impedances in the body [3].

\section{System Architecture}

We propose the architecture shown in Fig. 1 for canceling interference in an area efficient way. It exploits oversampling to reduce the size of the anti-aliasing filter and mixed-signal feedback to create a sharp, programmable notch. By using feedback, interferers are eliminated at the front end, relaxing the dynamic range requirements of the forward path components and enabling the use of a lower supply voltage. The motivation for exploiting oversampling arises from recent advances in ADC design that have led to ultra-low energy/conversionstep figures of merit [4]. This enables the use of oversampling without a significant power penalty. Using the $4.4 \mathrm{fJ} / \mathrm{step}$ FOM cited in [4], an 8-bit ADC sampling at $10 \mathrm{kS} / \mathrm{s}$ would

*The authors acknowledge the support of Texas Instruments, DARPA, and the Focus Center for Circuit \& System Solutions (C2S2), one of five research centers funded under FCRP, a Semiconductor Research Corporation Program.
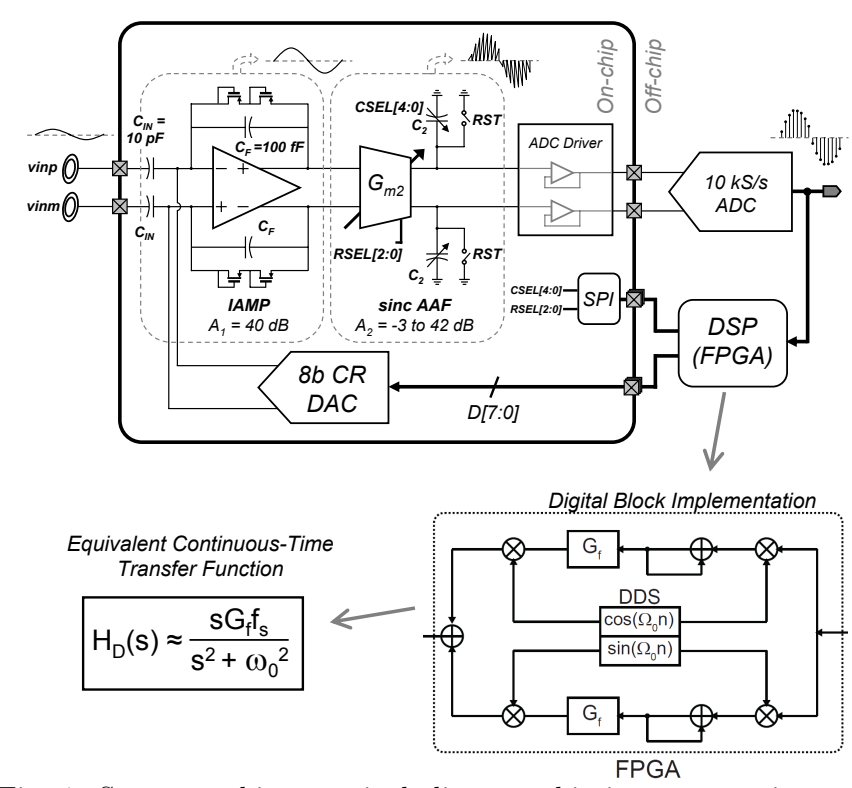

Fig. 1. System architecture including: on-chip instrumentation amplifier, charge-sampling sinc filter, and charge redistribution feedback DAC; and off-chip ADC and digital signal processor.

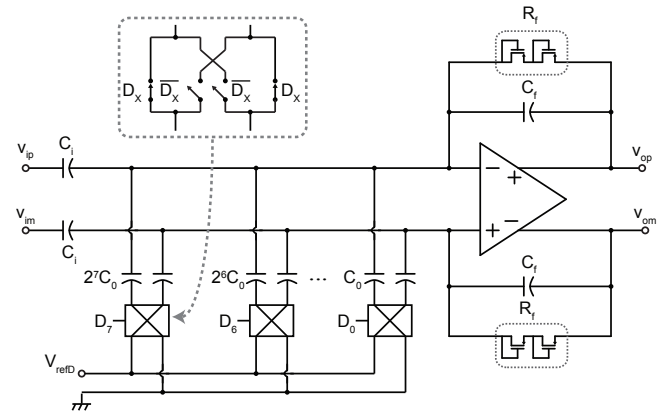

Fig. 2. Combined IAMP and charge redistribution feedback DAC.

only consume $11 \mathrm{nW}$; at least an order of magnitude less than a typical IAMP. For a bio-potential signal with a $100 \mathrm{~Hz}$ bandwidth, the oversampling ratio (OSR) of 50 results in a dynamic range of more than $65 \mathrm{~dB}$ which is better than typically required $(\mathrm{DR}=1.76 \mathrm{~dB}+6.02 \mathrm{~dB} \times \mathrm{ENOB}+10 \log (\mathrm{OSR}))$.

The IAMP in Fig. 1 is similar to [1], but fully differential to reduce the supply voltage requirements. A charge-sampling anti-aliasing filter with a sinc frequency response is used to accurately place notches at the aliasing frequencies while providing programmable gain [5]. Its transconductor's output current is integrated onto capacitors $C_{2}$ for period $T_{s}$, and at the end of each period, the resulting voltage is digitized by the ADC. The capacitors are then reset using the switches labeled $R S T$ and a new cycle begins. The resulting charge sampling yields a sinc frequency response with notches at integer multiples of $f_{s}=1 / T_{s}$. Since these are precisely the frequencies that would be aliased onto the discrete-time baseband, the filter inherently provides anti-aliasing. The transconductor is similar to [6]. The filter's gain is $A_{2}=2 G_{m 2} T_{s} / C_{2}$ and is digitally configured using switched resistors and capacitors.

\section{Mixed-Signal Feedback Loop}

The mixed-signal feedback loop used to cancel out PLI includes a forward path comprising: the IAMP, filter, ADC 


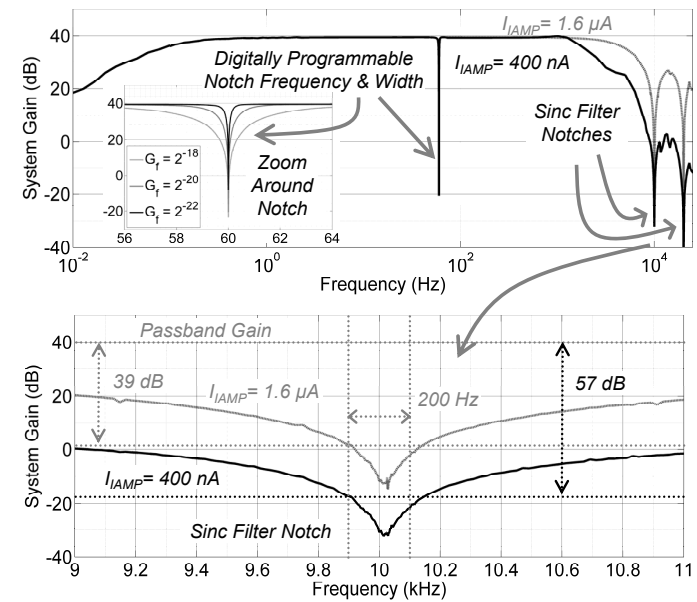

Fig. 3. Frequency response showing a sharp, programmable notch for interference cancelation and sinc filter notches for anti-aliasing.

driver, and ADC; and a feedback path comprising a digital block implemented on an FPGA and an 8-bit chargeredistribution DAC. The DAC, shown in Fig. 2, shares the IAMP's opamp, and therefore consumes only incremental $f C V^{2}$ power that is very small. The digital block uses a 32-bit direct digital synthesizer (DDS) to create digital sine and cosine signals with discrete-time frequency $\Omega_{0}$. The notch frequency of the closed-loop system is $\omega_{0}=f_{s} \Omega_{0}$ and can be set to any value between zero and $f_{s} / 2$ with steps as small as $3 \mu \mathrm{Hz}$ (for $f_{s}=10 \mathrm{kHz}$ ). The equivalent continuous-time transfer function of the ADC, digital block, and DAC combination, $H_{D}(s)$, is shown on the bottom left of Fig. 1. It has the form of an integrator, up-converted to $\omega_{0}$. The closed-loop response, therefore, has two sharp notches at $\pm \omega_{0}$. The notch width is set by $G_{f}$ and is also programmable using a digital register. Values of $G_{f}$ between $2^{-22}$ and $2^{-18}$ can be used to select notch widths between $0.5 \mathrm{~Hz}$ and $8 \mathrm{~Hz}$, respectively.

The system architecture in Fig. 1 is conceptually similar to [7], but by implementing the integrator digitally, the area and power are reduced many orders of magnitude. In [7], 10 $\mathrm{M} \Omega$ resistors and $100 \mu \mathrm{F}$ capacitors are used to create the long time constants necessary to achieve a sharp notch frequency. Our implementation achieves the required long time constants using digital registers that are area and power efficient.

\section{Measurements and Conclusion}

The top plot in Fig. 3 shows the full frequency response of the system. The IAMP has a $120 \mathrm{mHz}$ high pass corner frequency to filter out EDO. The gray trace shows the frequency response when the IAMP is biased with $1.6 \mu \mathrm{A}$ of current. This relatively high bias current extends the closed-loop bandwidth of the opamp beyond $25 \mathrm{kHz}$ such that the filter dominates the magnitude response. The sinc response notches are clearly seen at $10 \mathrm{kHz}$ and $20 \mathrm{kHz}$. For a lower bias setting of $400 \mathrm{nA}$, the IAMP provides some additional low-pass filtering. The bottom plot in Fig. 3 zooms in to the notch at $10 \mathrm{kHz}$ and shows that the filter provides more than $39 \mathrm{~dB}$ of anti-aliasing over $200 \mathrm{~Hz}$ of bandwidth and the IAMP provides an additional $18 \mathrm{~dB}$ when its bias current is set to $400 \mathrm{nA}$. The plot insert in Fig. 3 zooms in to the digitally programmable notch which was set to $60 \mathrm{~Hz}$ for this measurement. As shown, its bandwidth can be digitally set via $G_{f}$.

Depending on the thermal noise requirements, the IAMP bias current can be set anywhere between $100 \mathrm{nA}$ and 1.6 $\mu \mathrm{A}$. The filter's bias current is typically set to $50 \mathrm{nA}$. Since a $1.5 \mathrm{~V}$ supply was used, the combined power consumption of these two blocks is typically between $225 \mathrm{nW}$ and $2.5 \mu \mathrm{W}$. The
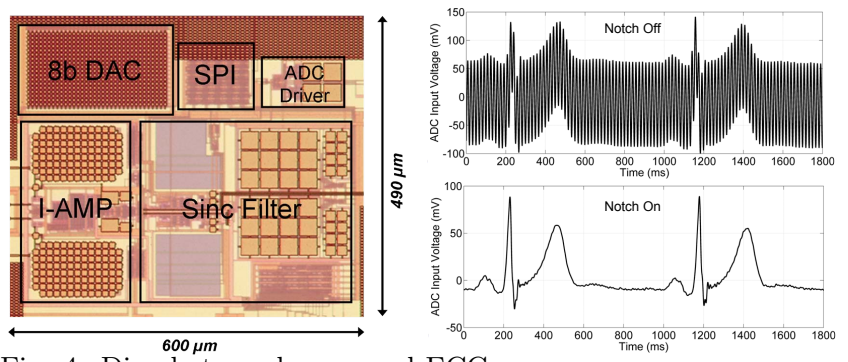

Fig. 4. Die photo and measured ECG.

feedback DAC consumes less than $30 \mathrm{nW}$.

Fig. 4 shows a die photograph of the chip and an ECG measurement performed with our system with and without using the notch. The combined area of the IAMP, DAC and filter is less than $0.225 \mathrm{~mm}^{2}$. The filter was made significantly larger than is necessary to allow for maximum flexibility. For $f_{s}=10 \mathrm{kHz}$, its gain, which is inversely proportional $R_{s}$ and $C_{2}$, can be tuned from $-3 \mathrm{~dB}$ to $42 \mathrm{~dB}$. For typical applications where the minimum gain can be set to $10 \mathrm{~dB}$ or more, the size of the filter can be reduced considerably.

For the ECG measurement, the input impedance of the IAMP was artificially degraded to exacerbate the effect of PLI and emulate a worst case scenario. As shown in the figure, when the notch filter is turned on, the $60 \mathrm{~Hz}$ interference is completely eliminated.

\begin{tabular}{|c|c|c|c|c|}
\hline & This Work & {$[1]$} & {$[2]$} & {$[7]$} \\
\hline $\mathrm{V}_{\text {supply }}$ & $1.5 \mathrm{~V}$ & $\pm 2.5 \mathrm{~V}$ & $1.8 \mathrm{~V}$ & $\pm 5 \mathrm{~V}$ \\
\hline $\mathrm{I}_{\text {supply }}$ & $370 \mathrm{nA}^{*}$ & $180 \mathrm{nA}$ & $1 \mu \mathrm{A}$ & Not Avail. \\
\hline $\mathrm{P}_{\text {supply }}$ & $555 \mathrm{nW}^{*}$ & $900 \mathrm{nW}$ & $1.8 \mu \mathrm{W}$ & Not Avail. \\
\hline Passband & $0.12-100 \mathrm{~Hz}^{*}$ & $0.01-30 \mathrm{~Hz}$ & $0.05-180 \mathrm{~Hz}$ & $0.05-150 \mathrm{~Hz}$ \\
\hline Noise & $3.4 \mu \mathrm{V}_{r m s}{ }^{*}$ & $1.6 \mu \mathrm{V}_{r m s}$ & $1.0 \mu \mathrm{V}_{r m s}$ & Not Avail. \\
\hline Gain & $37-82 \mathrm{~dB}$ & $39.8 \mathrm{~dB}$ & $41,50.5 \mathrm{~dB}$ & $55 \mathrm{~dB}$ \\
\hline Filter & $\operatorname{sinc}$ & N/A & $2-$ pole LPF & Discrete \\
\hline Notch & Mixed-Sig. & N/A & N/A & Analog \\
\hline Area & $0.225 \mathrm{~mm}^{2}$ & $0.220 \mathrm{~mm}^{2}$ & $1.7 \mathrm{~mm}^{2}$ & N/A \\
\hline \multicolumn{5}{|c}{} \\
\hline
\end{tabular}

TABLE I. Comparison with recent publications.

In conclusion, we present a fully differential biomedical sensor interface with a low-power IAMP, sinc anti-aliasing filter, and mixed-signal feedback for interference cancelation. The novel use of charge-sampling for anti-aliasing yields the added benefit of $45 \mathrm{~dB}$ of gain programmability without consuming excessive area. The digital implementation of the feedback path in the interference cancelation loop yields a flexible, areaefficient solution. Finally, by canceling interferers at the front end of the system, the dynamic range requirements of the forward path blocks are relaxed.

\section{References}

[1] R. R. Harrison et al., "A Low-Power IC for a Wireless 100-Electrode Neural Rec. Sys.," IEEE JSSC, Jan. 2007.

[2] T. Denison et al., "A $2 \mu \mathrm{W} 100 \mathrm{nV} / \sqrt{\mathrm{Hz}}$ ChopperStabilized Instrumentation Amplifier for Chronic Measurement of Neural Field Potentials," IEEE JSSC, Dec. 2007.

[3] J. C. Huhta and J. G. Webster, "60-Hz Interference in Electrocardiography," IEEE TBE, Mar. 1973.

[4] M. van Elzakker et al., "A $1.9 \mu \mathrm{W} 4.4 \mathrm{fJ} /$ Conv. step 10b $1 \mathrm{MS} / \mathrm{s}$ Charge Redist. ADC," IEEE ISSCC, Feb. 2008.

[5] G. Xu and J. Yuan, "Comparison of Charge Sampling and Voltage Sampling," IEEE MSSCC, Aug. 2000.

[6] D. R. Welland et al., "A Digital Read/Write Channel with EEPR4 Detection," ISSCC, Feb. 1994.

[7] I.-D. Hwang et al., "Direct Interference Canceling for TwoElectrode Biopotential Amplifier," IEEE TBE, Nov. 2008. 